\title{
Implicancias de la educación sexual en la formación docente
}

\author{
What sexual education involves in teacher education
}

María Victoria Plaza y Elsa Meinardi

Grupo de Didáctica de la Biología. Centro de Formación e Investigación en Enseñanza de las Ciencias. Facultad de Ciencias Exactas y Naturales, Universidad de Buenos Aires, Argentina. mariviplaza99@hotmail.com

\section{Resumen}

El trabajo que presentamos es parte de un proyecto de investigación que tiene como objetivo analizar las representaciones sociales sobre sexualidad que se evidencian en el llamado currículo oculto escolar. Para ello se reflexionará en forma conjunta con docentes de diversas materias del nivel secundario sobre dichas representaciones y se determinará su posible incidencia en las prácticas profesionales docentes y en la construcción de las representaciones de los alumnos.

En Argentina se han sancionado dos leyes nacionales, una de Salud Sexual y Procreación Responsable y otra de Educación Sexual Integral, por lo cual los/las docentes están obligados a enseñar Educación Sexual en las instituciones escolares. Es una temática difíal de abordar por lo aral creemos que se necesita un análisis profundo de las representaciones sociales sobre sexualidad. Esperamos con este proyecto aportar herramientas en este sentido.

\section{Palabras claves}

Educación sexual, representaciones, currículo oculto, sexualidad.

\section{Abstract}

This paper is part of a research Project which has the aim of analyzing representations of sexuality manifested in the so-called hidden curriculum. With this aim we will discuss these representations with secondary school teachers of different subjects and we will establish their likely incidence on the professional teacher practices and on the construction of the students' representations.

In Argentina two national acts were passed, one about Sexual Health and Responsible Procreation and the other about Integral Sexual Education, both establishing obligatory Sexual Education courses at school. Sex education remains a complex/controversial issue, that is why we think that deep analysis on the sexual representations is necessary. With this project we hope to provide some useful tools in this regard.

\section{Keywords}

Integral sexual education, representations, hidden curriaulum, sexuality 


\section{Introducción}

La Educación Sexual hoy

Actualmente en América Latina se considera que la educación sexual debe hacerse a partir de una acción de intencionalidad educativa, a través del desarrollo de una estrategia de enseñanza sistemática basada en la información y en el análisis de la misma con los alumnos y alumnas. Es decir, se trata de generar una conciencia en los y las jóvenes, respecto no solamente del uso de anticonceptivos, sino también del cuidado personal, del respeto por todas las personas sin discriminar su condición social, la elección de su pareja sexual o la elección de género, entre otras.

Con respecto a la educación sexual podemos decir que en Argentina existían - y aún existen en muchos casos - dos prácticas predominantes de educación sexual; una de ellas basada en la enseñanza, por parte del o la docente de biología, de contenidos que induían los métodos anticonceptivos, los sistemas reproductivos de varones $y$ mujeres $y$ la planificación familiar (Meinardi et al, 2008); otra práctica muy común consistúa en invitar a un profesional de la salud para que diera charlas sobre estos temas a los y las estudiantes. De esta manera se aumplía con la formalidad de "dar educación sexual". El mayor inconveniente de esta última es que una vez que el o la profesional se retira de la escuela no quedaba un adulto referente a quién consultar. Estas acciones, aunque bien intencionadas, están muy alejadas de la educación sexual mencionada al principio.

La nueva perspectiva acerca de la Educación Sexual se fue incorporando recientemente en algunas aulas por decisión de los y las docentes, pero luego de la sanción, en nuestro país en 2006, de la Ley Nacional de Educación Sexual Integral, el profesorado está obligado a tratar estos temas. La ley, por un lado legitima lo que, en algunos casos, ya se venía haciendo desde hace unos años, y por otro limita lo que se puede hacer y decir dentro de los contenidos referidos a la educación sexual. Anteriormente el profesorado podía informarse en estos temas si así lo deseaba, pero actualmente, como consecuencia de la ley, se encuentra en la obligación de hacerlo, ya que en ella se establece "que todos los educandos tienen derecho a recibir educación sexual integral en los establecimientos educativos públicos, de gestión estatal y privada de la Argentina".

Una educación sexual "integral" implica la articulación de aspectos biológicos, psicológicos, sociales, afectivos y éticos. Estos mismos elementos se encuentran en la construcción de la sexualidad humana, un proceso mediante el cual cada persona incorpora y elabora un conjunto de pautas, expectativas, conocimientos, creencias, valores, normas y actitudes que regulan el ejercicio de dicha sexualidad.

\section{Desarrollo}

\section{Educación Sexual y Formación Docente}

A grandes rasgos, cuando el/la docente se ocupa de la educación sexual en el aula, pueden suceder tres tipos de situaciones en las que se relacionan las propias concepciones 0 representaciones sobre sexualidad del docente con los contenidos a ser enseñados. Una de ellas ocurre cuando el/la docente no está de acuerdo, por distintos motivos, con los lineamientos auriculares nacionales (LCN) sancionados en 2008 bajo el marco de la ley antes mencionada; por ejemplo porque tiene una concepción biologicista de la educación sexual la cual se traduce en sólo transmitir información sobre biología y fisiología de la reproducción humana. Otra variante es aquella en la que el profesorado está de acuerdo con la prescripción curricular e intenta un tratamiento integral de la educación sexual, pero, al asumir el papel de "actor", es capaz de vislumbrar que sostiene algunos juicios de valor negativos en auestiones relacionadas con la sexualidad y el género; si bien esto podńa provocarle un conflicto entre sus concepciones y aquello que debe enseñar, al mismo tiempo tiene la ventaja de permitirle reflexionar acerca de ciertos 
elementos que integran su representación sobre la sexualidad y la educación sexual. Una tercera posibilidad, aunque estimamos que es la menos frecuente, se da cuando el tratamiento que el o la docente hace en el aula coincide y es legitimado con la prescripción curricular, en cuyo caso no se genera tal conflicto.

Lo anteriormente mencionado pone daramente en evidencia que los procesos de educación sexual en la escuela requieren de una formación de manera que lo que ocurre en el aula durante el tratamiento de estos temas, no quede librado al "voluntarismo". Y más aún, hace pensar también en la formación que reciben los profesores sobre el tema. La ley de Educación Sexual Integral tiene puesta la mirada en la educación sexual de niños, niñas y adolescentes de la Argentina. Pero los que deben impartir dicha educación son los propios maestros y maestras, por lo aual la mirada también está puesta en ellos/as. La educación sexual integral que se plantea trae como consecuencia una transformación de la cultura; esta transformación no responderá simplemente a una modificación cognitiva, sino que debería implicar una modificación en la dimensión moral y ética de las personas. Esto propone un cambio en la estructura misma de la educación inicial y media, y en los desafíos de la formación decente, poniendo el acento en el marco institucional en que ésta se lleva a cabo. Los Lineamientos Curriculares Nacionales le proponen al docente los contenidos que debe tratar en sus aulas y la forma en que debe enseñar educación sexual (de manera integral). De esta forma se le pide al maestro/a que su mirada frente a la sexualidad sea integral y sin prejuicios, pero no se los/las ha formado para ello. Araujo (2005) menciona que las Universidades y centros de formación en educación superior son responsables de la formación de estos sujetos, hombres y mujeres, que estanían llamados a producir una transformación cultural actuando desde el ámbito educativo.

Pacheco Silva y Pacheco de Carvalho (2005) plantean que cada grupo de alumnos posee diferentes tabúes y prejuicios relacionados con la sexualidad, lo cual exige al docente un mayor conocimiento de los contenidos del tema y de las estrategias didácticas que se necesitan para cada grupo, ya que los contenidos de educación sexual deben ir siendo modificados según las necesidades de los/las alumnos/as. Las autoras mencionan en su investigación que las docentes encuestadas expresan la necesidad de estar bien con su propia sexualidad para hablar de estos temas con sus alumnos/as. Es una necesidad impuesta por el mismo contenido para evitar en el aula que el/la docente asuma posturas personales cuando se están discutiendo temas polémicos. Es decir, el/la docente que trata la educación sexual en el aula debería rever sus concepciones o representaciones sobre sexualidad.

En relación a lo antes mencionado el educador/educadora que trabaja en educación sexual debe tener daro que realiza una tarea a dos puntas: una externa hacia los demás y una interna hacia sí mismo/a. Si bien su objetivo fundamental es trabajar sobre las "actitudes" de sus alumnos/as, permanentemente pone en juego sus propias representaciones o concepciones sobre la sexualidad, y al mismo tiempo, sobre la educación sexual deseable; de allí que van a signar su actuación frente al estudiantado.

Dichas concepciones o representaciones constituyen una modalidad de pensamiento práctico, es decir, una actividad mental orientada hacia la práctica, un principio que sirve de guía para la actuación concreta sobre los seres humanos y sobre las cosas (Jodelet, 2003). La función de las mismas es la elaboración de los comportamientos y la comunicación entre los individuos, es el conocimiento de sentido común que tiene como objetivo comunicar, estar al día y sentirse dentro del ambiente social, y que se origina en el intercambio de comunicaciones del grupo social.

En líneas generales se podría afirmar que las representaciones constituyen modalidades de pensamiento práctico orientados hacia la comunicación, la comprensión y el dominio del entomo social, material e ideal. Este pensamiento de sentido común se constituye a partir de las experiencias de cada persona, de la información, los conocimientos y modelos de pensamiento que 
se obtienen, y que también se trasmiten, por medio de la tradición, la educación y la comunicación social. De este modo, este conocimiento es, en muchos aspectos, un conocimiento socialmente elaborado y compartido.

En nuestro trabajo nos interesa concentramos en aquellas situaciones, antes mencionadas, en las que el o la docente se sitúa -voluntariamente o de manera no explícita para él mismo- en una posición de conflicto entre aquello que debe enseñar y sus propias concepciones, porque consideramos que dichas concepciones o representaciones van a estar presentes en el denominado curríaulo oculto escolar, formando parte fundamental de aquello que se enseña y, por qué no, compitiendo con el curríalo prescripto o dedarado por el profesorado.

\section{Concepciones y Currículo Oculto}

Se denomina currículo oculto a aquello que se enseña de manera implícita en las instituciones escolares y de lo que no se acostumbra a hablar en las dedaraciones de fines y objetivos de los/las profesores/as. Dicho currículo oculto se revela sobretodo en las ausencias, las omisiones, las jerarquizaciones, las contradicciones y los desconocimientos que presentan el currículo oficial denominado formal- y la práctica diaria docente que, se supone, lo realiza (Mosconi, 1998); lo que se considera el currículo real.

En el trabajo que presentamos, el aual es parte de un proyecto de tesis doctoral, nos proponemos investigar las representaciones sobre sexualidad que presentan los y las docentes de una escuela media de la ciudad de Buenos Aires que van a conformar el currículo oculto escolar. Abordamos este trabajo desde una perspectiva didáctica, en la cual las investigaciones sobre representaciones se nutren de diversas fuentes, de las cuales nos centraremos fundamentalmente en los aportes de la psicología evolutiva; de la epistemología, con los modelos sobre obstáculos (desarrollados por Bachellard y posteriormente por Astolfi y otros) y de la psicología social, con las representaciones sociales de Moscovici.

La metodología del trabajo involucra observaciones de dase no participantes, un período de reflexión dialógica entre investigador y docentes con el fin de hacer explícitos algunos elementos de dichas representaciones -que caracterizaremos- para finalmente determinar su posible incidencia en las prácticas profesionales docentes y en la construcción de las representaciones sobre sexualidad de los alumnos y alumnas.

\section{Conclusión}

Dentro del gran número de investigaciones que existen actualmente sobre el currículo oculto escolar, no hemos encontrado aquellas que se relacionen directamente con las concepciones de sexualidad de los y las docentes, sino que se basan, en general, en las concepciones de género.

Nuestras investigaciones hasta el momento muestran que las representaciones que se evidencian en dicho curníaulo son las más difíciles de modificar, ya que al ser implícitas se manifiestan de manera involuntaria dentro del vocabulario escolar sin que se tome conciencia de ellas. Lo comprometido de estas representaciones es que algunas muestran signos de discriminación, en relación con el género, la orientación sexual o el comportamiento de los y las jóvenes. Trabajamos bajo la hipótesis de que el desocultar las representaciones sobre sexualidad de los docentes permitirá reconocer y tal vez modificar lo que están aprendiendo los y las alumnas de manera implícita.

Una característica importante de este proyecto es que los y las docentes (del grupo investigado) reflexionarán sobre su práctica diaria escolar y también sobre la formación que han recibido. Como mencionan Aliau y Duschatzky (2003) en la medida en que se reconozca el carácter arbitrario del 
modelo de educación sexual que se presenta como natural, y en tanto se visualice como producción social, podrá cuestionarse, explicarse y modificarse el llamado currículo oculto. Por eso consideramos importante reflexionar sobre los saberes implíatos puestos en juego en la práctica escolar.

\section{Bibliografía}

Alliau, A. y Duschatzky, L (comp.) (2003) Maestros. Formación, práctica y transformación escolar: Buenos Aires: Niño y Dávila Editores

Araujo, K. (2005). La construcción de una práctica pedagógica sensible a la problemática de género. Los desafíos de la formación y las prácticas docentes. Chile: Seminario Internacional Equidad de Género en las Reformas Educativas de América Latina -17 y 18 de mayo.

Jodelet, D. (2003). Entrevista a Denis Jodelet por Óscar Rodríguez Cerda. México: Relaciones, invierno, 24 (93), 115-134

Meinardi E., Godoy E., Iglesias M., Revel Chion A., Rodríguez Vida I., Plaza M V., Bonan L (2008) Educación para la Salud Sexual en la formación de profesores en Argentina, Ciencia \& Educacao, 14 (2), 181-195

Mosconi, N. (1998). Diferencia de sexos y su relación con el saber: Buenos Aires: Colección Formación de formadores, Ediciones Novedades Educativas

Pacheco Silva, M. y Pacheco de Carvalho, W. L (2005) O desenvolvimiento do conhecimento pedagógico di conteúdo de sexualidade na vivencia das professoras, Ciencia \& Educacao, 11 (1), 73-82 